\title{
Association between oral contraceptive use as a risk factor and triple-negative breast cancer: A systematic review and meta-analysis
}

\author{
LI LI, YAHUA ZHONG, HONGYAN ZHANG, HAIJUN YU, YONG HUANG, \\ ZHENG LI, GAILI CHEN and XINYING HUA
}

Department of Radiation and Medical Oncology, Zhongnan Hospital, Wuhan University, Wuhan, Hubei 430071, P.R. China

Received April 13, 2016; Accepted March 6, 2017

DOI: $10.3892 /$ mco.2017.1259

\begin{abstract}
Triple-negative breast cancer (TNBC), a unique subtype of breast cancer, which is resistant to endocrine and targeted therapy, usually relapses early, progresses rapidly and is associated with a poor prognosis. Epidemiological investigations focusing on the association between risk factors and the onset of TNBC demonstrated that the incidence of TNBC exhibits a significant correlation with anthropometric, geographical and demographic parameters. The aim of the present systematic review and meta-analysis was to evaluate the strength of the association between the use of oral contraceptives (OCs) and TNBC. Two databases (PubMed Central/PubMed, Web of Science) and secondary references were searched to identify studies meeting the priorly established inclusion criteria. Case-control studies published between January, 2005 and March, 2016 were searched using the key words (triple-negative breast cancer OR basal-like) AND (oral contraceptives). Finally, 9 eligible articles using as control other subtypes of invasive breast cancer and 7 articles using a healthy population as control were incorporated in the meta-analysis. Pooled odds ratio (OR) and $95 \%$ confidence interval (CI) were calculated using fixed- or random-effects models according to the heterogeneity between studies. The case-control comparison using other subtypes of breast cancer as the control arm exhibited a significant association between OC use and TNBC (OR $=1.31,95 \% \mathrm{CI}=1.18-1.45 ; \mathrm{Z}=5.26$, $\mathrm{P}<0.00001)$. These results were further confirmed by the case-control comparison using the healthy population as the control $\operatorname{arm}(\mathrm{OR}=1.21,95 \% \mathrm{CI}=1.01-1.46 ; \mathrm{Z}=2.04, \mathrm{P}=0.04)$. The present meta-analysis indicated that women who use OCs have a greater risk of TNBC compared with women who
\end{abstract}

Correspondence to: Dr Yahua Zhong, Department of Radiation and Medical Oncology, Zhongnan Hospital, Wuhan University, 169 Donghu Road, Wuchang, Wuhan, Hubei 430071, P.R. China E-mail: yahua_zhong@163.com

Key words: triple-negative breast cancer, oral contraceptives, risk factor, molecular marker do not. This conclusion prompts that women who used OCs should be examined more closely in population screenings of breast cancer, as they may benefit from prevention and early detection strategies.

\section{Introduction}

Breast cancer is one of the most common malignant tumors in women and the leading cause of death among middle-aged women (1). Breast cancer may be classified into several subtypes according to its different etiology, clinicopathological presentation, molecular characteristics and response to therapy. Molecular biological methods are considered to be the gold standard for their accuracy in diagnosis due to the nature of the specific biological characteristic of the different breast cancer subtypes (2). In general, estrogen receptor (ER), progesterone receptor (PR) and human epidermal growth factor receptor 2 (HER2) are used as markers for invasive breast cancer classification in order to predict the outcome and select the optimal therapeutic strategies for the management of breast cancer (3).

With the development of molecular biology technology in recent years, an increasing number of molecular markers may be selected to classify the subtypes of invasive breast cancer. Due to the complexity, cost and lack of uniform standards of gene chip technology, it is difficult to apply in clinical practice. In clinical practice, immunohistochemistry for determining the ER, PR and HER2 status has been used to classify breast cancer into four subtypes, namely luminal A, luminal B, HER2-overexpressing and triple-negative breast cancer (TNBC) (4).

TNBC is a subtype of breast cancer, in which the expression of all three molecular markers is negative, and accounts for $10-20 \%$ of all breast cancers. TNBC has a higher mortality and risk of metastasis compared with other subtypes of breast cancer $(5,6)$. In addition, it is highly invasive and is associated with a high local recurrence risk, and poor cancer-specific and disease-free survival (7-9).

The reasons for the low survival rate of TNBC are mainly as follows: First, compared with other subtypes of breast cancer, TNBC is associated with a higher rate of chromosomal mutations, high mitotic count, mutation of the p53 and BRCA1 
genes, and lymphatic dissemination $(10,11)$. Second, TNBC cannot be accurately diagnosed using conventional imaging examinations and is often only detected at an advanced stage (12). Third, therapies such as hormone therapy, targeted therapy and chemotherapy have not been shown to be effective (12).

Previous studies have demonstrated that there is a strong association between TNBC and race. TNBC accounts for $12-17 \%$ of all breast cancers in Western populations (13), whereas its incidence is higher $(\leq 50 \%)$ in African-American women. The incidence in Asian women is similar to that in Caucasian women, and BRCA1 mutations are more common in these patients $(11,14)$. Previous findings have also demonstrated that obese women are at a greater risk of developing TNBC compared with non-obese women (15). The use of oral contraceptives (OCs) has been identified as an important factor in the development of breast cancer (16), but there are currently no studies clearly indicating an association between OCs and TNBC. Moreover, the established risk factors for breast cancer as a whole may not apply to this unique subgroup of patients.

The aim of the present systematic review and meta-analysis was to focus on case-control studies and elucidate the strength of the association between OCs and TNBC, enabling a better understanding of TNBC, helping clinicians integrate biological and epidemiological characteristics, providing a theoretical basis and more information for epidemiology researchers focusing on the etiology, prevention and treatment of TNBC.

\section{Materials and methods}

Literature search and criteria for eligible studies. The PubMed Central/PubMed and Web of Science databases were systematically searched between January, 2005 and March, 2016 using the search terms (triple-negative breast cancer OR basal-like) AND (oral contraceptives). In addition, the reference lists of the studies were manually searched for related articles. Two authors (L.L. and Y.Z.) screened the research results independently according to priorly established inclusion criteria.

Based on the history of OC use, patients were classified as non-OC users or OC users. If a patient had used OCs for $>1$ year, she was classified as an $\mathrm{OC}$ user.

Case-control studies reporting the status of ER, PR and HER2 as detected by immunohistochemistry or molecular biology methods were included in the final analysis. Additional molecular makers (cytokeratin 5/6, epidermal growth factor receptor) were analyzed by certain studies, but were not used in the present analysis. TNBCs, characterized by absence of expression of all three markers, were assigned to the case arm. Patients who expressed one or more of the abovementioned markers were assigned to the control arm. When a healthy population was used in the articles, it was assigned to another control arm. Therefore, the control group included other subtypes of breast cancer patients or healthy individuals for different groups of analysis.

The following inclusion criteria were established to minimize bias or heterogeneity in the selection of studies: i) Full-text articles published between January, 2005 and March, 2016; ii) case-control studies; iii) data on the correlation between $\mathrm{OC}$ use and TNBC; iv) original articles written in
English; v) diagnosis of TNBC implemented by immunohistochemistry stain or molecular biology methods; vi) original data included in the articles were sufficient to calculate the odds ratio (OR) and 95\% confidence interval (CI); vii) at least 20 patients included in the case group; and viii) if there was an overlap in the cases included, only the latest and most comprehensive data were selected. Any disagreements were resolved through discussion.

Data extraction and quality evaluation. The following information was independently extracted from the original studies by two reviewers: Name of the first author, year of publication, research location, histological type of the tumors, sample size of the case and control groups, and correction factor. In order to guarantee the methodological quality of the included studies, the literatures were required to meet the following basic criteria: The diagnosis of TNBC should be definitive based on immunohistochemistry or molecular biological methods and the major confounding factors should be specified and analyzed. Two reviewers independently assessed the methodological quality of the included studies according to the Newcastle-Ottawa scale. Any disagreements were arbitrated by discussion and majority voting of all authors.

Data synthesis and statistical analysis. In order to analyze the association between OC use and TNBC, the pooled ORs and 95\% CIs were calculated by the original data in the studies. The results indicated that OCs may cause TNBC with a high probability if OR $>1$, compared with non-use of OCs. Heterogeneity among studies was evaluated using the Chi-squared test and $\mathrm{I}^{2}$ statistic. If the P-value of the Chi-squared test was $<0.1$, the heterogeneity was considered to be significant (17). The $\mathrm{I}^{2}$ test may be quantified in the evaluation of study heterogeneity (the greater the $\mathrm{I}^{2}$, the greater the heterogeneity among studies). Under normal conditions, if the $\mathrm{I}^{2}$ value was $<50 \%$, the heterogeneity was not considered to be significant. Heterogeneity may be decreased by the following methods: i) Subgroup analysis; and ii) sensitivity analysis of the test results of potential bias. When the Chi-squared test and the $\mathrm{I}^{2}$ statistic indicate that heterogeneity is not significant, the pooled OR value may be calculated using the Mantel-Haenszel fixed-effects model; otherwise, the DerSimonian-Laird random-effects model is adopted (14). A funnel plot was used to evaluate the potential publication bias. Data synthesis and statistical analysis were performed using RevMan v.5.3 software (International Cochrane Collaboration Network, Copenhagen, Denmark) and the CI was set to $95 \%$.

\section{Results}

Study selection. A total of 392 articles were initially identified from the database search, and 11 case-control studies met the inclusion criteria and were included in the final meta-analysis. Ultimately, 9 studies were included in the group using other subtypes of breast cancer as the control arm, and 7 studies were included in the quantitative analysis using the healthy population as the control arm. A total of 15,427 patients participated and 3,279 were diagnosed with TNBC. The characteristics and original data of the studies included in the case-control analysis are summarized in Table I. 
Table I. Characteristics of the studies included in the meta-analysis.

\begin{tabular}{lcccc}
\hline Authors & Study year & Data source & Control types & Refs. \\
\hline Beaber et al & $2004-2010$ & United States & $\mathrm{H}$ & $(18)$ \\
Bethea et al & $1993-2001,1995-2015,2003-2015$ & BWHS, CBCS, WCHS & $\mathrm{H}$ & $(19)$ \\
Dolle et al & $1983-1990,1990-1992$ & SEER & H, P & $(20)$ \\
Lee et al & $1998-2003$ & WHI & P & $(21)$ \\
Kabat et al & $1993-1998,1998-2005$ & WHI & H, P & $(22)$ \\
Kwan et al & $1997-2000,2006-2009$ & LACE & P & $(23)$ \\
Ma et al & $1994-1998$ & United States & H, P & $(24)$ \\
Kawai et al & $2004-2010$ & CSS & H, P & $(25)$ \\
Lara-Medina et al & $1998-2008$ & Hispanic & P & $(26)$ \\
Millikan et al & $1993-1996,1996-2001$ & CBCS & P & $(27)$ \\
Phipps et al & $1993-1998$ & WHI & H, P & $(28)$ \\
\hline
\end{tabular}

WHI, Women's Health Initiative; CBCS, Carolina Breast Cancer Study; WCHS, Women's Circle of Health Study; BWHS, Black Women's Health Study; LACE, Life After Cancer Epidemiology; CSS, Cancer Surveillance System; SEER, Seattle-Puget Sound Surveillance, Epidemiology, and End Results; H, healthy population; P, patients.

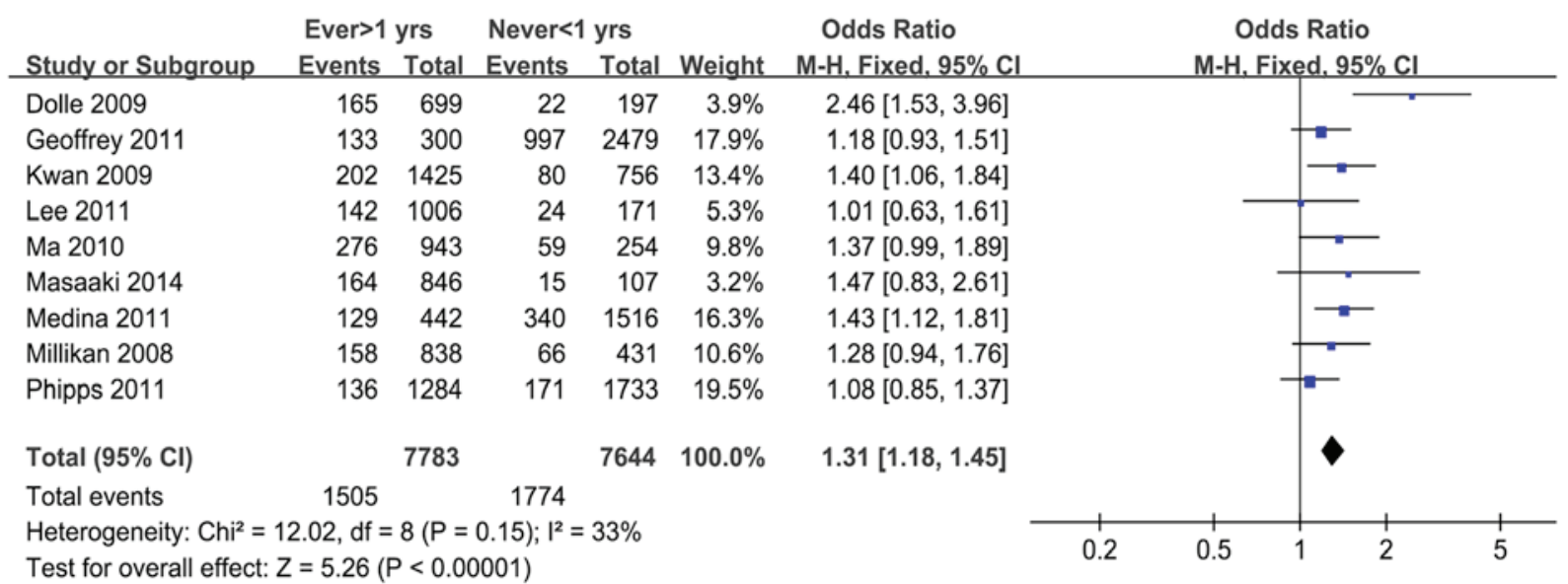

Figure 1. Forest plot with 95\% CI for individual and pooled ORs for case-control studies in TNBC and non-TNBC, where the control arm was other subtypes of breast cancer. CI, confidence interval; OR, odds ratio; TNBC, triple-negative breast cancer.

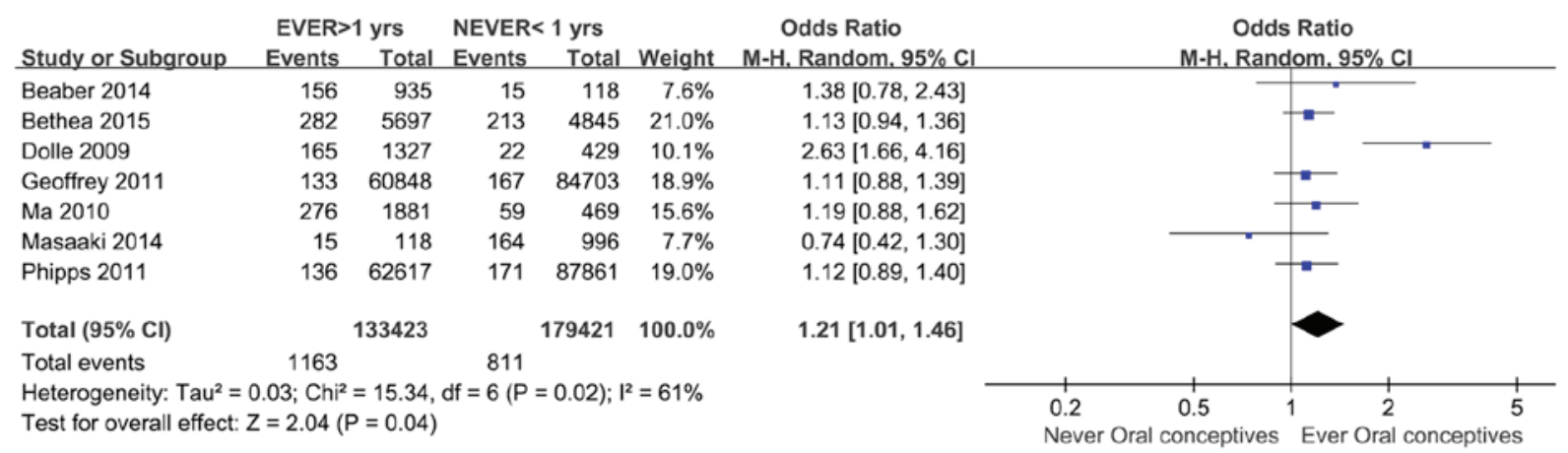

Figure 2. Forest plot with 95\% CI for individual and pooled ORs for case-control studies in TNBC and non-TNBC, where the control arm was the healthy population. CI, confidence interval; OR, odds ratio; TNBC, triple-negative breast cancer.

When all the data were synthesized, no significant heterogeneity was detected. For the studies using other subtypes of breast cancer as the control arm, heterogeneity was not significant $\left(\mathrm{P}=0.15, \mathrm{I}^{2}=33 \%\right)$. These results demonstrated that $\mathrm{OC}$ use increased the risk of TNBC among these women $($ pooled $\mathrm{OR}=1.31,95 \% \mathrm{CI}=1.18-1.45 ; \mathrm{Z}=5.26$, 


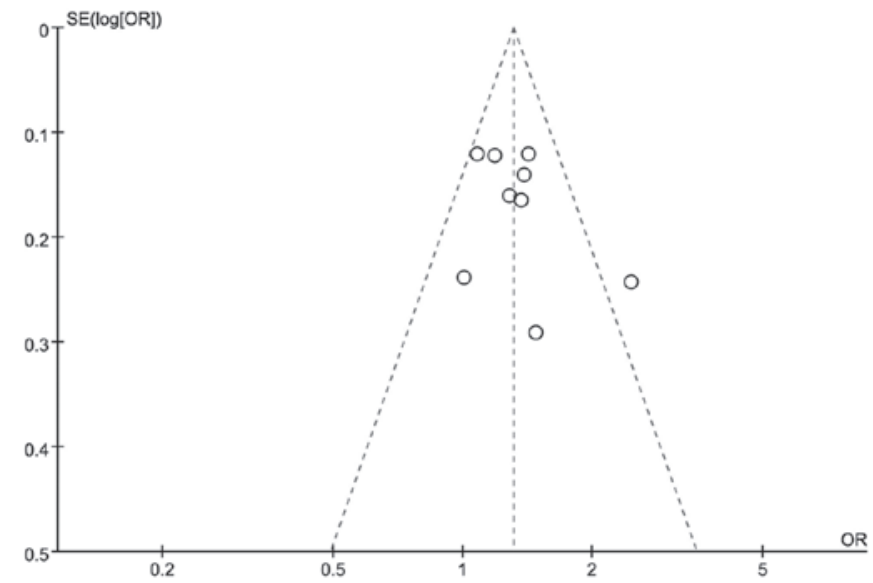

Figure 3. Funnel plot with $95 \%$ CI for evaluating the publication bias for the pooled OR in studies using other subtypes of breast cancer as control. CI, confidence interval; OR, odds ratio; SE, standard error.

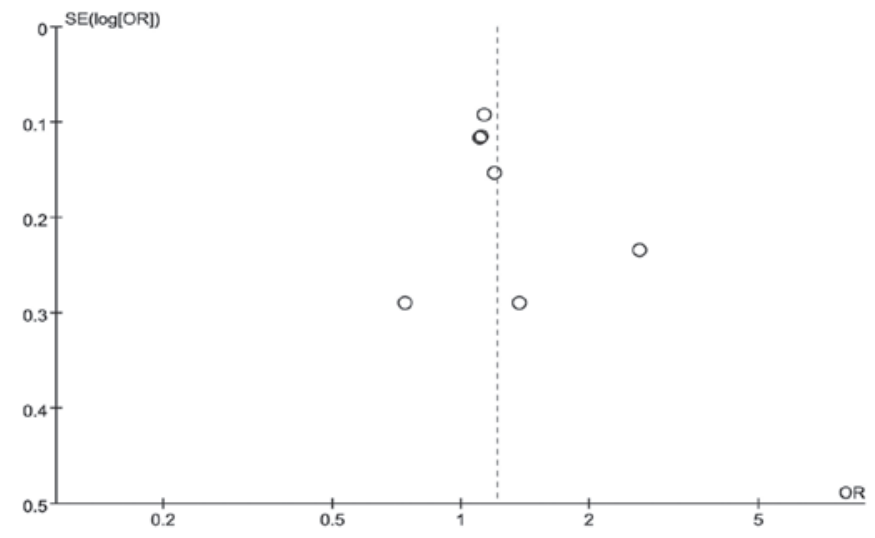

Figure 4. Funnel plot with $95 \%$ CI for evaluating the publication bias for the pooled OR in studies using the healthy population as control. CI, confidence interval; OR, odds ratio; SE, standard error.

$\mathrm{P}<0.00001$ ) (Fig. 1). Using data from healthy individuals as the control arm, the association between OC use and TNBC was further confirmed $(\mathrm{OR}=1.21,95 \% \mathrm{CI}=1.01-1.46$; $\mathrm{Z}=2.04, \mathrm{P}=0.04$ ) (Fig. 2).

Publication bias was assessed by funnel plots (Figs. 3 and 4); the data were distributed in the left and right parts of the graph, and there was no obvious asymmetry. This demonstrated that publication bias was not significant, and the study heterogeneity was unlikely due to publication bias.

\section{Discussion}

Previous studies indicated that the use of OCs may increase the risk of TNBC; however, the conclusions of these studies were conflicting. Indeed, Dolle et al (20), Beaber et al (18) and Kwan et al (23) reported that OC use increases the risk of TNBC; however, Marchbanks et al (29), Kawai et al (25) and Kabat et al (22) did not confirm this association.

The onset of TNBC is affected by multiple factors. The results of this analysis demonstrated that $\mathrm{OC}$ use increases the risk of TNBC. This result suggested that i) the pathological mechanism underlying OC use increasing the risk of TNBC requires further investigation; and ii) women who use OCs should be more closely followed regarding TNBC screening and prevention. It was previously demonstrated that TNBC was associated with family history $(30,31)$, race $(32,33)$, obesity $(15,34)$ and menopausal status $(35)$. These conclusions indicated that TNBC exhibits a certain association with hormone status. OCs are likely to exert a certain effect on hormone levels; thus, it is reasonable to infer that OC use is a likely risk factor for TNBC, with several hypotheses explaining this phenomenon. One of the mechanisms through which OC use affects breast cancer in younger women is the combination of estrogen and ER positivity in the mammary gland developing cancer, which promotes tumor growth and angiogenesis. The second mechanism is that estrogen increases the density of vascular distribution and recruitment of stromal cells systematically, which promotes tumor growth in $\mathrm{ER}^{-}$as well as $\mathrm{ER}^{+}$cases (36). The second mechanism may play a leading role in breast carcinogenesis.

The present meta-analysis analyzed the strength of association between OC use and TNBC, compared with non-TNBC cases or the healthy population. The non-TNBC control group demonstrated that $\mathrm{OC}$ use may cause an increase in the risk of TNBC, and the healthy population further confirmed this conclusion.

The present systematic review and meta-analysis had several limitations. First, the number of TNBC cases was relatively small and the history of OC use was dichotomous. This classification is relatively crude, and cannot explain the association between exposure dose of OCs and TNBC. Second, the use of hormone replacement therapy was not controlled in this analysis. Therefore, the population under investigation may have obvious endocrinology heterogeneity. In order to obtain a more accurate strength of association between TNBC and $\mathrm{OC}$ use, this analysis requires more studies to appropriately control the abovementioned potential confounding factors. In addition, the study classified the use of OCs into never or ever users, whereas more detailed information such as types or dose of OCs was not taken into consideration. Because of the relatively limited number of studies and heterogeneous information obtained by different analyses, information on confounding factor control and heterogeneity are difficult to obtain.

Even with these limitations, the present study provides a new perspective on the association between TNBC and physiological and pathological conditions. In order to ensure the accuracy of the analysis, a funnel plot was used to analyze the publication bias of the included studies, with the results showing that publication bias was not significant.

The identification of risk factors for TNBC may provide a reference for the screening and prevention of the target population. Overall, the results of this meta-analysis indicate that women who use OCs are more likely to develop TNBC compared with women who do not. Women who use OCs, particularly combined with other risk factors of TNBC, should be followed more closely. In addition, the mechanism underlying the increased risk of breast cancer, such as TNBC, also requires further investigation.

The results of the present study should draw more attention to the disease screening and follow-up of TNBC patients and the use of OCs, particularly as risk factors of TNBC. 


\section{Acknowledgements}

The present study was supported by a grant from the Outstanding Scientific Fund of Wuhan University (no. 2042014kf0162).

\section{References}

1. Jemal A, Siegel R, Ward E, Hao Y, Xu J, Murray T and Thun MJ: Cancer statistics, 2008. CA Cancer J Clin 58: 71-96, 2008.

2. Rakha EA and Ellis IO: Triple-negative/basal-like breast cancer: review. Pathology 41: 40-47, 2009.

3. Huo D, Ikpatt F, Khramtsov A, Dangou JM, Nanda R, Dignam J, Zhang B, Grushko T, Zhang C, Oluwasola O, et al: Population differences in breast cancer: survey in indigenous African women reveals over-representation of triple-negative breast cancer. J Clin Oncol 27: 4515-4521, 2009.

4. Lv M, Li B, Li Y, Mao X, Yao F and Jin F: Predictive role of molecular subtypes in response to neoadjuvant chemotherapy in breast cancer patients in Northeast China. Asian Pac J Cancer Prev 12: 2411-2417, 2011.

5. Dawood S, Lei X, Litton JK, Buchholz TA, Hortobagyi GN and Gonzalez-Angulo AM: Impact of body mass index on survival outcome among women with early stage triple-negative breast cancer. Clin Breast Cancer 12: 364-372, 2012.

6. De Giorgi U, Rosti G, Frassineti L, Kopf B, Giovannini N, Zumaglini F and Marangolo M: High-dose chemotherapy for triple negative breast cancer. Ann Oncol 18: 202-203, 2007.

7. Brady-West DC and McGrowder DA: Triple negative breast cancer: therapeutic and prognostic implications. Asian Pac J Cancer Prev 12: 2139-2143, 2011.

8. Choi J, Jung WH and Koo JS: Clinicopathologic features of molecular subtypes of triple negative breast cancer based on immunohistochemical markers. Histol Histopathol 27: 1481-1493, 2012.

9. Vaklavas C and Forero-Torres A: How do I treat 'triple-negative' disease. Curr Treat Options Oncol 12: 369-388, 2011.

10. de Ruijter TC, Veeck J, de Hoon JP, van Engeland M and Tjan-Heijnen VC: Characteristics of triple-negative breast cancer. J Cancer Res Clin Oncol 137: 183-192, 2011.

11. Irvin WJ Jr and Carey LA: What is triple-negative breast cancer? Eur J Cancer 44: 2799-2805, 2008.

12. Foulkes WD, Smith IE and Reis-Filho JS: Triple-negative breast cancer. N Engl J Med 363: 1938-1948, 2010.

13. Brenton JD, Carey LA, Ahmed AA and Caldas C: Molecular classification and molecular forecasting of breast cancer: ready for clinical application? J Clin Oncol 23: 7350-7360, 2005.

14. Stang A: Critical evaluation of the Newcastle-Ottawa scale for the assessment of the quality of nonrandomized studies in meta-analyses. Eur J Epidemiol 25: 603-605, 2010.

15. Pierobon $M$ and Frankenfeld CL: Obesity as a risk factor for triple-negative breast cancers: a systematic review and meta-analysis. Breast Cancer Res Treat 137: 307-314, 2013.

16. Rosenberg L, Zhang Y, Coogan PF, Strom BL and Palmer JR: A case-control study of oral contraceptive use and incident breast cancer. Am J Epidemiol 169: 473-479, 2009.

17. Sutton AJ, Abrams KR, Jones DR, Sheldon TA and Song F: Methods for meta-analysis in medical research. In: Statistics in Medicine. Schwarzer and Guido (eds). Vol 22. John Wiley \& Sons, Inc., pp3112-3114, 2003.

18. Beaber EF, Malone KE, Tang MT, Barlow WE, Porter PL, Daling JR and Li CI: Oral contraceptives and breast cancer risk overall and by molecular subtype among young women. Cancer Epidemiol Biomarkers Prev 23: 755-764, 2014

19. Bethea TN, Rosenberg L, Hong CC, Troester MA, Lunetta KL, Bandera EV, Schedin P, Kolonel LN, Olshan AF, Ambrosone CB and Palmer JR: A case-control analysis of oral contraceptive use and breast cancer subtypes in the African American Breast Cancer Epidemiology and Risk Consortium. Breast Cancer Res 17: 22, 2015.

20. Dolle JM, Daling JR, White E, Brinton LA, Doody DR, Porter PL and Malone KE: Risk factors for triple-negative breast cancer in women under the age of 45 years. Cancer Epidemiol Biomarkers Prev 18: 1157-1166, 2009.
21. Lee E, McKean-Cowdin R, Ma H, Spicer DV, Van Den Berg D, Bernstein L and Ursin G: Characteristics of triple-negative breast cancer in patients with a BRCA1 mutation: results from a population-based study of young women. J Clin Oncol 29: 4373-4380, 2011

22. Kabat GC, Kim M, Phipps AI, Li CI, Messina CR, Wactawski-Wende J, Kuller L, Simon MS, Yasmeen S, WassertheilSmoller S and Rohan TE: Smoking and alcohol consumption in relation to risk of triple-negative breast cancer in a cohort of postmenopausal women. Cancer Causes Control 22: 775-783, 2011.

23. Kwan ML, Kushi LH, Weltzien E, Maring B, Kutner SE, Fulton RS, Lee MM, Ambrosone CB and Caan BJ: Epidemiology of breast cancer subtypes in two prospective cohort studies of breast cancer survivors. Breast Cancer Res 11: R31, 2009.

24. Ma H, Wang Y, Sullivan-Halley J, Weiss L, Marchbanks PA, Spirtas R, Ursin G, Burkman RT, Simon MS, Malone KE, et al: Use of four biomarkers to evaluate the risk of breast cancer subtypes in the women's contraceptive and reproductive experiences study. Cancer Res 70: 575-587, 2010.

25. Kawai M, Malone KE, Tang MTC and Li CI: Active smoking and the risk of estrogen receptor-positive and triple-negative breast cancer among women ages 20 to 44 years. Cancer 120: 1026-1034, 2014

26. Lara-Medina F, Pérez-Sánchez V, Saavedra-Pérez D, Blake-Cerda M, Arce C, Motola-Kuba D, Villarreal-Garza C, González-Angulo AM, Bargalló E, Aguilar JL, et al: Triple-negative breast cancer in Hispanic patients: high prevalence, poor prognosis, and association with menopausal status, body mass index, and parity. Cancer 117: 3658-3669, 2011.

27. Millikan RC, Newman B, Tse CK, Moorman PG, Conway K, Dressler LG, Smith LV, Labbok MH, Geradts J, Bensen JT, et al: Epidemiology of basal-like breast cancer. Breast Cancer Res Treat 109: 123-139, 2008.

28. Phipps AI, Chlebowski RT, Prentice R, McTiernan A, Wactawski-Wende J, Kuller LH, Adams-Campbell LL, Lane D, Stefanick ML, Vitolins M, et al: Reproductive history and oral contraceptive use in relation to risk of triple-negative breast cancer. J Natl Cancer Inst 103: 470-477, 2011.

29. Marchbanks PA, Curtis KM, Mandel MG, Wilson HG, Jeng G, Folger SG, McDonald JA, Daling JR, Bernstein L, Malone KE, et al: Oral contraceptive formulation and risk of breast cancer. Contraception 85: 342-350, 2012.

30. Tariq K and Rana F: TNBC vs. non-TNBC: a five-year retrospective review of differences in mean age, family history, smoking history and stage at diagnosis at an inner city university program. World J Oncol 4: 241-247, 2013.

31. Phipps AI, Buist DSM, Malone KE, Barlow WE, Porter PL, Kerlikowske K and Li CI: Family history of breast cancer in first-degree relatives and triple-negative breast cancer risk. Breast Cancer Res Treat 126: 671-678, 2011.

32. Rosenberg L, Boggs DA, Wise LA, Adams-Campbell LL and Palmer JR: Oral contraceptive use and estrogen/progesterone receptor-negative breast cancer among African American women. Cancer Epidemiol Biomarkers Prev 19: 2073-2079, 2010.

33. Trivers KF, Lund MJ, Porter PL, Liff JM, Flagg EW, Coates RJ and Eley JW: The epidemiology of triple-negative breast cancer, including race. Cancer Causes Control 20: 1071-1082, 2009.

34. Phipps AI, Malone KE, Porter PL, Daling JR and Li CI: Body size and risk of luminal, HER2-overexpressing, and triple-negative breast cancer in postmenopausal women. Cancer Epidemiol Biomarkers Prev 17: 2078-2086, 2008.

35. Rose DP and Vona-Davis L: Interaction between menopausal status and obesity in affecting breast cancer risk. Maturitas 66: 33-38, 2010

36. Gupta PB, Proia D, Cingoz O, Weremowicz J, Naber SP, Weinberg RA and Kuperwasser C: Systemic stromal effects of estrogen promote the growth of estrogen receptor-negative cancers. Cancer Res 67: 2062-2071, 2007. 